\title{
The Influence of Culture Conditions on Carotenogenesis in Streptococcus faecium UNH564P
}

\author{
By R. F. TAYLOR AND B. H. DAVIES \\ Department of Biochemistry and Agricultural Biochemistry, \\ University College of Wales, Aberystwyth, Dyfed, SY23 3 DD \\ (Received 3 July 1975; revised 3 September 1975)
}

\begin{abstract}
S U M MAR Y
The growth of Streptococcus faecium UNH564P and its production of triterpenoid carotenoids under a variety of culture conditions were examined. Total extractable cell lipid and carotenoid levels increased with culture age and paralleled the growth curve of the bacterium. Variations of the medium glucose concentration produced significant changes in both cell growth and carotenoid production, with the xanthophyll content decreasing at high glucose concentrations. Carotenoid degradation products were found in highly aerated cultures although a high glucose concentration appeared to have a sparing effect on oxidative degradation. Culture age appeared to have little effect on carotene:xanthophyll ratios. The significance of the production of total and individual carotenoids under the various culture conditions is discussed and related to a postulated scheme of triterpenoid carotenoid biosynthesis in the organism.
\end{abstract}

\section{INTRODUCTION}

In the past, the effects of variations in a number of parameters on carotenoid formation in micro-organisms have been investigated. Culture age and growth stage influence not only the total amount of carotenoid but also the specific type of carotenoid present. In general, carotenes (hydrocarbon carotenoids) are present in the greatest concentration during early growth stages while xanthophylls (oxygenated carotenoids), if these are present at all, predominate in the late-exponential and stationary phases of growth. Thus in the photosynthetic bacterium Rhodopseudomonas palustris, the major carotenoid changes from

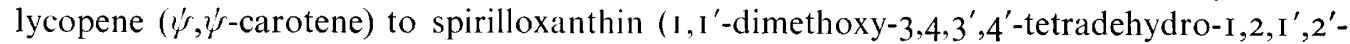
tetrahydro- $\psi^{\prime}, \psi^{\prime}$-carotene) as the culture ages from 6 days to 6 weeks (Goodwin, 1956). In Rhodospirillum rubrum, the carotenes reach a maximum concentration at $24 \mathrm{~h}$ but are not detectable at 72 to $96 \mathrm{~h}$; spirilloxanthin reaches its maximum concentration in 72 to $96 \mathrm{~h}$ (van Niel, Goodwin \& Sissins, 1956).

The composition of the culture medium also influences carotenogenesis. In Acholeoplasma laidlawii the carotenoid content of the plasma membrane can be increased by growing the cells in a sodium acetate-containing medium or decreased by using a medium containing propionate, thallium acetate or diphenylamine (Razin \& Cleverdon, 1965; Razin \& Rottem, 1967). The total carotenoid content of Epicoccum nigrum Link was found to vary markedly as a result of changes in the medium glucose concentration; maximum carotenoid concentration resulted from a total depletion of glucose (Foppen, 1969). Other studies on the effects of nutritional factors on carotenogenesis in micro-organisms have been reported in detail in a number of reviews (e.g. Goodwin, 1952, 1955, I97I).

In identifying the carotenoids of the non-photosynthetic Gram-positive bacterium, 
Streptococcus faecium UNH564P, we characterized a new class of pigments, the triterpenoid $\left(C_{30}\right)$ carotenoids (Taylor \& Davies, I 974 $\left.a, b, 1976\right)$. The series includes a number of both carotenes and xanthophylls which appear to be related by a biosynthetic sequence (Fig. 3) proceeding through the carotenes to the major xanthophyll, 4-D-glucopyranosyloxy-4,4'diaponeurosporene (4-D-glucopyranosyloxy-4,4'-diapo- $7^{\prime}, 8^{\prime}$-dihydro- $\psi^{\prime} \%, \psi^{\prime}$-carotene). During structural studies on these compounds, the effects of varying such parameters as glucose concentration, degree of aeration, and the time of harvesting on the carotenoid composition of $S$. faecium UNH564P were investigated, and are the subject of the present paper.

\section{METHODS}

Organism. The bacterium used was Streptococcus faecium UNH564P (Taylor \& Davies, I $974 a$ ) and was obtained from Dr W. R. Chesbro, Department of Microbiology, University of New Hampshire, Durham, New Hampshire, U.S.A.

Cultures. The bacteria were grown statically in $40 \mathrm{l}$ batches in Io 1 flat-bottomed flasks at $37^{\circ} \mathrm{C}$ in the light $(3200 \mathrm{~lx})$. Shake cultures were grown in 21 conical flasks, each containing I 1 medium, at $37{ }^{\circ} \mathrm{C}$ in the light and at $200 \mathrm{rev}$. $\mathrm{min}$ in an orbital shaker. For both types of culture, the basal aqueous medium contained the individual components of Trypticase soy broth (Difco) supplemented with yeast extract and phosphate (Taylor \& Davies, 1974a). Its composition was as follows $(\%, \mathrm{w} / \mathrm{v})$ : Bacto-tryptone (Difco), $1 \cdot 7$; Bacto-soytone (Difco), 0.3; NaCl, $5 ; \mathrm{K}_{2} \mathrm{HPO}_{4}, 0.25 ; \mathrm{NaH}_{2} \mathrm{PO}_{4}, 0.16 ; \mathrm{Na}_{2} \mathrm{HPO}_{4}$, 0.09; yeast extract (Difco), O.I. When necessary, the $\mathrm{pH}$ was adjusted to 6.8 with $\mathrm{I} \mathrm{M}-\mathrm{NaOH}$ before sterilization. For investigating the effect of glucose concentration on the production of individual carotenoids, the above medium was supplemented with $0 . \mathrm{I}, 0.25$ or $0.5 \%(\mathrm{w} / \mathrm{v})$ glucose.

Batch cultures were inoculated with a $5 \%(\mathrm{v} / \mathrm{v})$ inoculum of a $24 \mathrm{~h}$ culture of $S$. faecium which had been grown in medium of the same composition as that to be inoculated. Bacteria were harvested from batch cultures with a Sharples continuous-flow centrifuge, washed once with $0.9 \%(\mathrm{w} / \mathrm{v})$ aqueous $\mathrm{NaCl}$ solution, once with water, and frozen at $-20{ }^{\circ} \mathrm{C}$ to await carotenoid extraction.

Determination of extinction, bacterial dry weight and $p H$. The extinctions of cultures were determined using a Klett-Summerson colorimeter (Arthur H. Thomas Co., Philadelphia, Pennsylvania, U.S.A.) fitted with a No. $54(520$ to $580 \mathrm{~nm})$ filter. Culture $\mathrm{pH}$ was determined (Pye Model $75 \mathrm{pH}$ meter) on the supernatant solutions resulting from cell harvesting. Determinations of bacterial dry weight were made as reported previously (Taylor \& Davies, 1974a).

Extraction and purification of carotenoids. Methods for solvent purification and for the extraction and purification of carotenoids from $S$. faecium have been described (Taylor \& Davies, 1974a, $b, 1976$ ). The washed cells collected from batch cultures were repeatedly extracted until colourless by homogenization in $4 \%(\mathrm{w} / \mathrm{v}) \mathrm{KOH}$ in methanol. The resulting extracts were combined, I vol. water was added and the mixture was repeatedly extracted with $0.5 \mathrm{vol}$. diethyl ether until the aqueous methanol phase was colourless. The bulked ether extract was washed to neutrality with water, dried over anhydrous $\mathrm{Na}_{2} \mathrm{SO}_{4}$ and concentrated to dryness in vacuo in a tared vessel. After weighing (extracted lipid, Table I), the residue was redissolved, if required, in Io $\mathrm{ml}$ diethyl ether and the $E_{435}$ of this solution used as an approximate measure of extracted carotenoid (Fig. I).

Individual carotenoids were isolated by column chromatography. The extracted lipid was dissolved in a small volume of diethyl ether and applied to a column $(25 \times 2 \mathrm{~cm})$ of CF-I I cellulose (Whatman Biochemicals Ltd, Maidstone, Kent) which had been pre- 
washed and packed using diethyl ether (Taylor \& Davies, I974b). Carotenes and nonglycosidic xanthophylls were eluted with diethyl ether or $2 \%(\mathrm{v} / \mathrm{v})$ acetone in ether while the more polar glycosidic carotenoid, 4-I)-glucopyranosyloxy-4,4'-diaponeurosporene, was recovered by elution with $25 \%(\mathrm{v} / \mathrm{v})$ acetone in ether. The solution containing the carotenes and non-glycosidic xanthophylls from the cellulose column was concentrated in vacuo to dryness and the residue redissolved in a small volume of light petroleum (b.p. 40 to $60 \mathrm{C})$ and applied to a column $(25 \times 2 \mathrm{~cm})$ of alumina (M. Woelm, Eschwege, Germany; neutral, Brockmann activity grade II) which had been pre-washed and packed using light petroleum. The chromatogram was developed with light petroleum followed by increasing concentrations $\left(0.25\right.$ to $\left.25^{\circ}: \mathrm{o}, \mathrm{v} / \mathrm{v}\right)$ of acetone in light petroleum; $25 \mathrm{ml}$ fractions were collected and examined spectrophotometrically and those having the same electronic absorption spectra and chromatographic behaviour were combined. The separated carotenoids were further purified on smaller columns $(15 \times 1 \mathrm{~cm})$ of alumina (neutral, grade II) developed with light petroleum containing increasing concentrations of acetone until thin-layer chromatographic purity (Taylor \& Davies, 1974a,b, 1976) was achieved.

Identification and quantitative determination of carotenoids. The purified carotenes and xanthophylls were identified by their electronic absorption spectra, column elution sequence and thin-layer chromatographic behaviour on comparison with well-authenticated standards (Taylor \& Davies, 1974a,b, 1976). Electronic absorption spectra were recorded from solutions of carotenoids in light petroleum (diethyl ether in the case of the glucoside). Accurate quantitative determinations were performed spectrophotometrically on solutions of known volume using the specific extinction coefficients $\left(E_{1 \mathrm{~m}}^{1 \%}\right)$ reported previously (Taylor \& Davies, 1974a,b); these were (in light petroleum): $4,4^{\prime}$-diapophytoene $\left(4,4^{\prime}\right.$ -

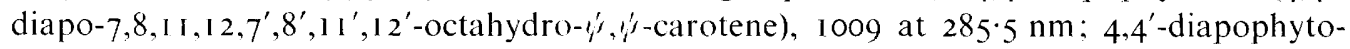
fluene $\left(4,4^{\prime}\right.$-diapo-7,8, II, 12, $7^{\prime}, 8^{\prime}$-hexahydro- $y^{\prime}, y^{\prime}$-carotene), 2 I05 at $346 \cdot 5 \mathrm{~nm}: 4,4^{\prime}$-diapo$7,8,11,12$-tetrahydrolycopene $\left(4,4^{\prime}\right.$-diapo-7,8, 1 1, I 2 -tetrahydro- $y^{\prime}, \psi$-carotene $), 3367$ at

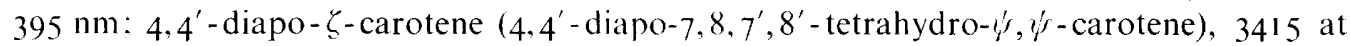
$400 \mathrm{~nm}$. The specific extinction coefficient of all-trans $4,4^{\prime}$-diaponeurosporene $(4,4$ '-diapo7,8-dihydro- $1 \%, \%$-carotene; 3905 at $435 \mathrm{~nm}$ in light petroleum) was also used as a nominal value in determining the amounts of ('is-isomers (neo B and neo C) of 4,4'-diaponeurosporene as well as the quantities of all the xanthophylls and carotenoid degradation products present. As the major carotenoids of $S$. faecium all have the 4,4 -diaponeurosporene chromophore (Taylor \& Davies, $1974 a, b$ ), changes in the amount of total extracted carotenoid could be deduced (but only in Fig. 1, which refers to a preliminary experiment) by following the $E_{435}$ of the total lipid extract.

\section{RISUITS}

\section{Effect of time on growth and lipid production}

To determine the effect of time on the growth and lipid content of S. faecium, fifteen 21 conical flasks, each containing I I of the standard medium with a $0.25 \%$ glucose supplement and inoculated from the same $24 \mathrm{~h}$ starter culture, were incubated in the light at $37^{\circ} \mathrm{C}$. At various times from o to $48 \mathrm{~h}$, the extinction and $\mathrm{pH}$ of the contents of one randomlyselected flask were determined, the cells harvested and the weight of extracted lipid and its $E_{435}$ determined. The values recorded in this way for culture $\mathrm{pH}$ and extinction differed by no more than $1 \%$ from those of a single control culture which was monitored throughout the experiment at the times chosen for harvesting the individual experimental flasks.

Exponential growth occurred during the period $\mathrm{I}$ to $\mathrm{I} 2 \mathrm{~h}$ after inoculation. The amount of 


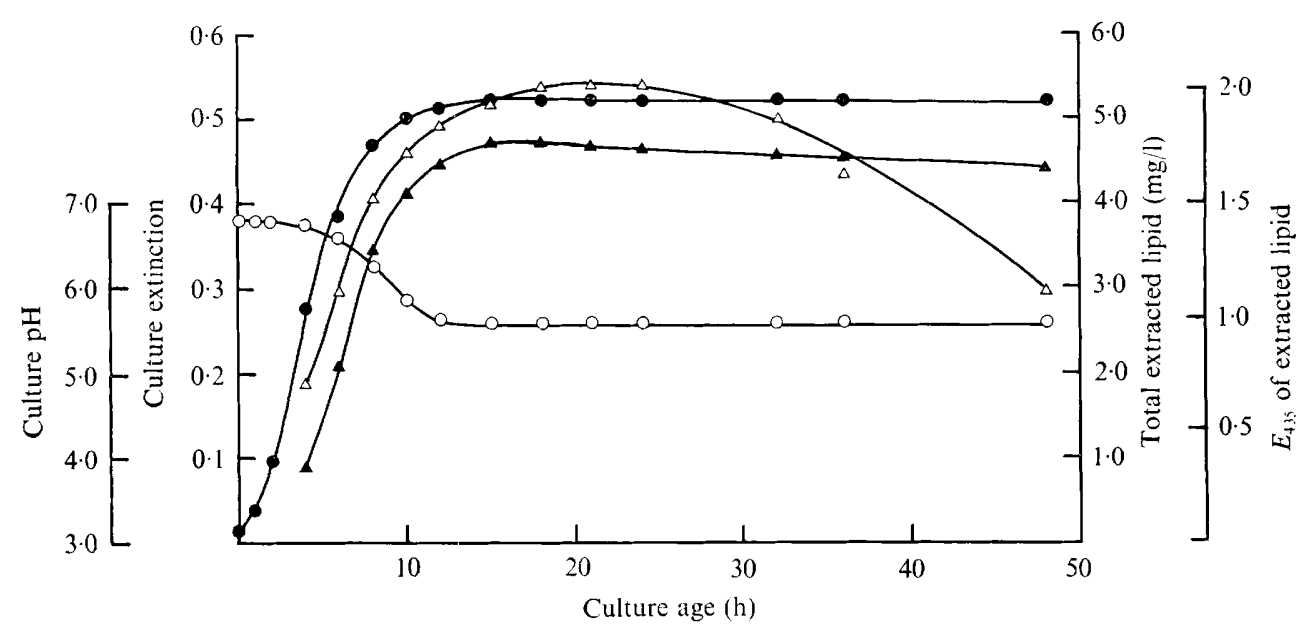

Fig. I. Growth as followed by culture extinction ( $\bigcirc)$, culture $\mathrm{pH}(\bigcirc)$ and levels of total extractable lipid $(\triangle)$ over a period of $48 \mathrm{~h}$ in static cultures of $S$. faecium grown on the basal medium supplemented with $0.25 \%(\mathrm{w} / \mathrm{v})$ glucose. Values for the $E_{435}$ of the extracted lipid $(\boldsymbol{\Delta})$ indicate the total carotenoid levels.

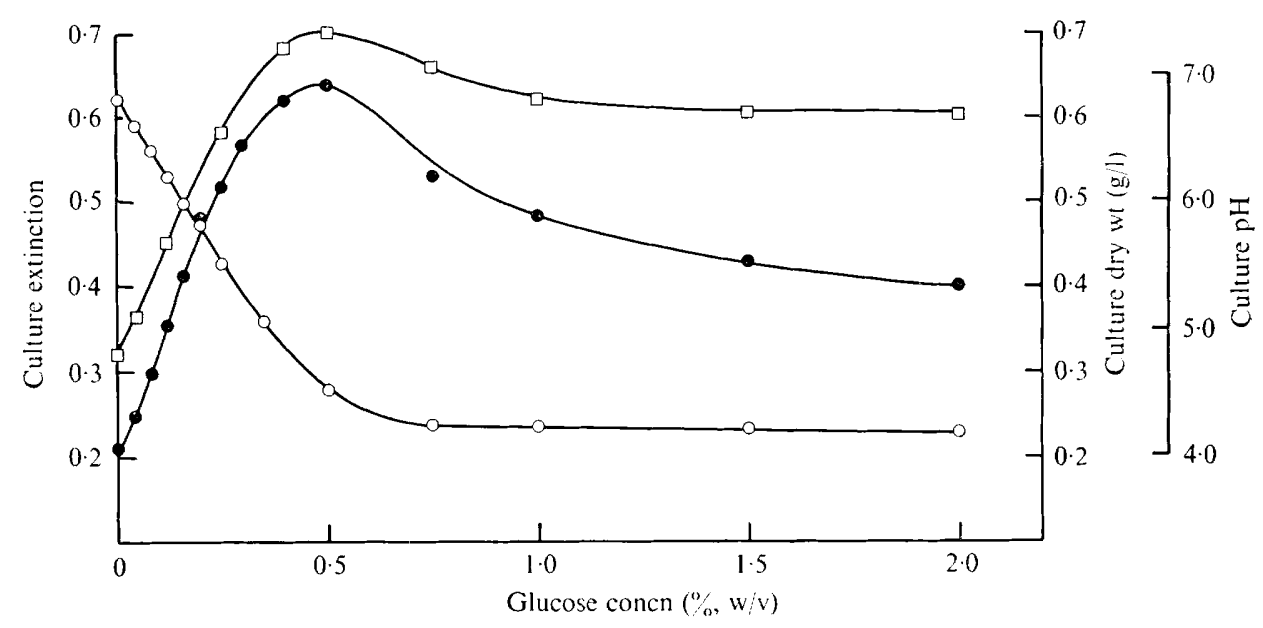

Fig. 2. Growth as indicated by culture extinction ( $O$ ) and culture dry weight $(\square)$, and culture pH (C) attained in $24 \mathrm{~h}$ by static cultures of $S$. faecium in the presence of different concentrations of glucose.

extractable lipid paralleled the growth curve but reached a maximum during the stationary phase (at $2 \mathrm{I} \mathrm{h}$ ) and then decreased to $53 \%$ of its maximum by the time the experiment terminated at $48 \mathrm{~h}$ (Fig. I). The $E_{435}$ values of the lipid extracts (indications of the amount of carotenoid present) also paralleled the growth curve, reached a maximum at $\mathrm{I} 8 \mathrm{~h}$, and then decreased slightly (by only $3 \%$ of their maximum value) until $48 \mathrm{~h}$. The pH of the cultures decreased from 6.8 at $\mathrm{o} h$ to 5.7 at $12 \mathrm{~h}$, and then remained constant. 


\section{Effect of glucose concentration on growth}

The effects of glucose concentration on growth were investigated in an experiment in which duplicate series of $50 \mathrm{ml}$ conical flasks, each containing $25 \mathrm{ml}$ basal medium supplemented with a different concentration of glucose (o to $2.0 \%$, w/v), were inoculated from a sterile water suspension of cells. These had been grown on a medium with a $0.25 \%$ glucose supplement for $24 \mathrm{~h}$, after which time they were centrifuged and washed twice with sterile distilled water. The experimental cultures were maintained in the light at $37^{\circ} \mathrm{C}$ for $24 \mathrm{~h}$, and then the extinction and $\mathrm{pH}$ of each culture, and the cell dry weights at ten selected glucose concentrations, were determined.

Cell growth (measured by culture extinction and cell dry weight) increased with increasing glucose concentrations up to $0.50 \%$, but thereafter decreased rapidly up to $\mathrm{I} \cdot 0 \%$ glucose and then more slowly (Fig. 2). Growth appeared to be related to medium $\mathrm{pH}$, which decreased with increasing glucose concentration but reached a steady value $(4 \cdot 2)$ at glucose concentrations above $0.75 \%$. The growth-limiting effect was presumably due to the buffering capacity of the medium being exceeded by an increased production of acid at high glucose levels.

\section{Effect of glucose concentration on carotenoid formation}

To study the effect of glucose concentration on carotenoid biosynthesis detailed quantitative comparisons were made (Tables I and 2) of the carotenoids formed in 24 and $48 \mathrm{~h}$ by 401 batches of cells growing at three selected glucose concentrations, namely $0.10,0.25$ and $0.50 \%(\mathrm{w} / \mathrm{v})$.

The lipid content of the cells showed a marked variation with different culture conditions (Table I). The highest amount of lipid found in static cultures was in those grown in the presence of $0.25 \%$ glucose for $24 \mathrm{~h}$, while the least amount was in those grown for $48 \mathrm{~h}$ on a medium containing the $0.10 \%$ glucose supplement. Quantitative analyses of the carotenoids of static cultures (Table I) showed the highest concentration to be present after $48 \mathrm{~h}$ growth in the $0.50 \%$ glucose medium, while the lowest was again in the cultures containing $0.10 \%$ glucose and grown for $48 \mathrm{~h}$. When the levels of carotenes and xanthophylls were compared (Table I), it was clear that carotene formation was higher at both $0 \cdot 10$ and $0.50 \%$ glucose than it was at $0.25 \%$ glucose; there was little difference in the relative carotene and xanthophyll levels of 24 and $48 \mathrm{~h}$ cultures at the same glucose concentrations.

Estimations of individual carotenoids purified from the cell lipid extracts (Table 2) revealed that three carotenoids predominated. These were 4,4'-diapophytoene, 4,4'diaponeurosporene and the major xanthophyll, 4-D-glucopyranosyloxy-4,4'-diaponeurosporene. As the glucose concentration increased from 0.10 to $0.50 \%$, the proportion of $4,4^{\prime}$-diapophytoene in the total carotenoid decreased from 85 to $3 \mathrm{I} \%$; there were concomitant increases in the levels of all the geometric isomers of $4,4^{\prime}$-diaponeurosporene, the proportion of all-trans $4,4^{\prime}$-diaponeurosporene increasing, for example, from $2 \cdot 3$ to $4 \mathrm{I} \%$ of the total carotenoid in $24 \mathrm{~h}$ cultures. In static cultures, the glucoside attained its highest level in those containing $0.25 \%$ glucose and fell to its lowest at $0.50 \%$ glucose.

The changes in these and all the other carotenes and xanthophylls supported the generalization that, in static cultures of $S$. faecium, less xanthophylls are formed at the expense of carotenes as the glucose concentration increases or decreases from $0.25 \%$. At a glucose concentration of $0.50 \%$, the major pigment is 4,4 -diaponeurosporene, the postulated carotene precursor of all the triterpenoid xanthophylls of $S$. faecium. 


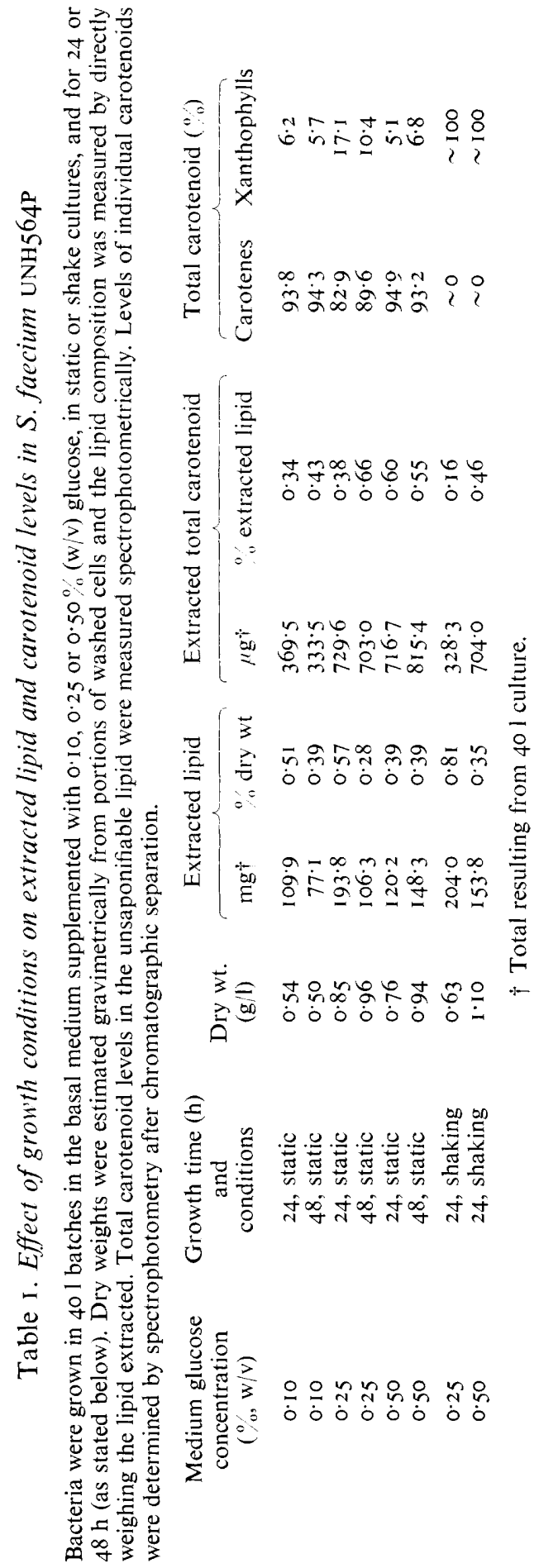




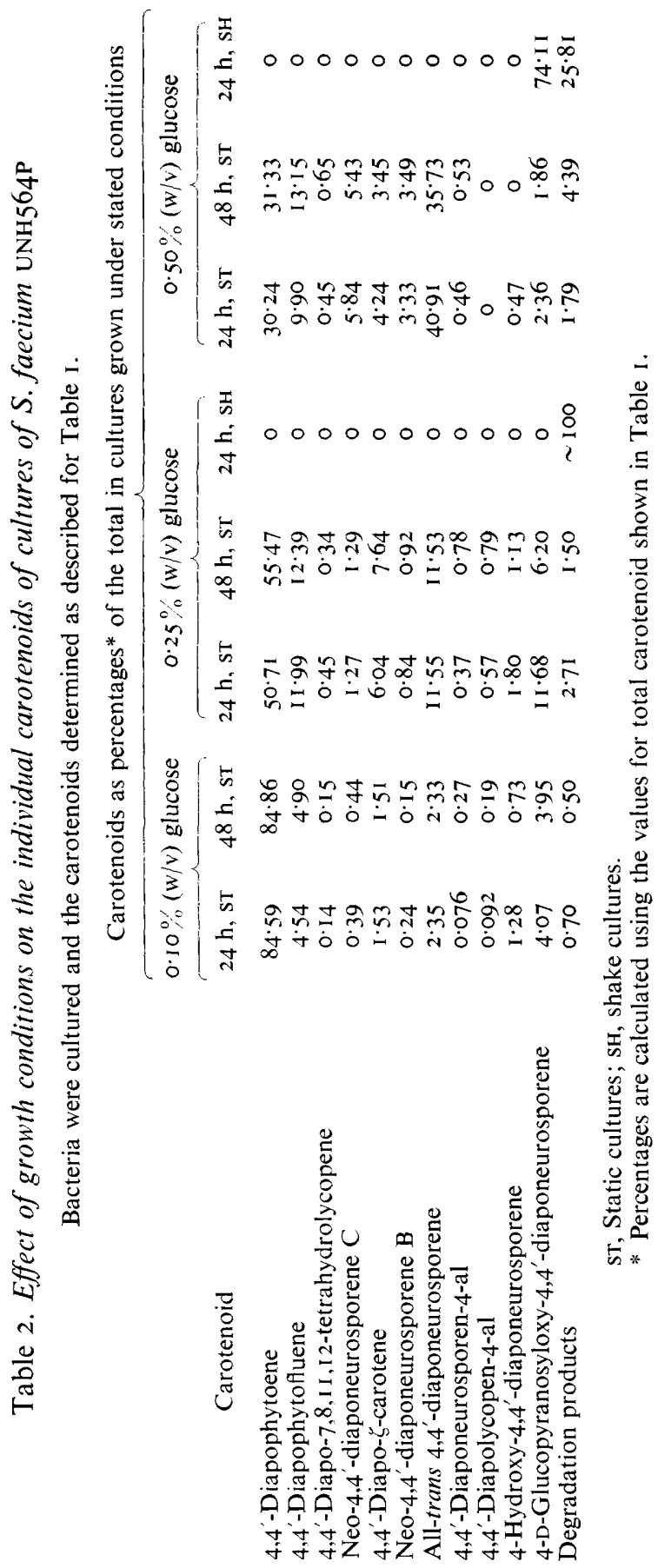




\section{Effect of shaking on carotenoid formation and degradation}

At an early stage in these investigations, it was observed that cells harvested from shake cultures were of a deeper yellow colour than those from static cultures. The phenomenon was investigated in more detail in an experiment in which lipid and carotenoid analyses were made of cells grown for $24 \mathrm{~h}$ in shake culture on media containing 0.25 and $0.50 \%$ glucose; the results of these analyses were compared (Tables I and 2) with those of cells grown in static culture under otherwise identical conditions.

The highest lipid concentration and lowest carotenoid content observed in all our studies were those of cells in shake cultures on the medium supplemented with $0.25 \%$ glucose. Nearly all the pigments present were xanthophylls (Table I), and these included (Table 2) only the glucoside and what were assumed to be carotenoid degradation products. The latter were compounds which were eluted from cellulose and alumina with only the most polar of solvents, and which had multi-peaked electronic absorptions corresponding to none of the known carotenoid spectra. In the $0.25 \%$ glucose shake cultures, nearly all the pigment consisted of such degraded material, while the glucoside was still the major carotenoid (comprising $74 \%$ of the total) in the $0.50 \%$ glucose shake cultures.

It therefore appears that under conditions of increased aeration, carotenoid production by $S$. faecium is directed primarily towards the formation of xanthophylls (which are then oxidatively degraded) and, at high glucose concentrations, to the formation of 4-D-glucopyranosyloxy- $4,4^{\prime}$-diaponeurosporene. Under the latter conditions, the formation of the glucoside has a sparing effect on carotenoid degradation.

\section{DISCUSSION}

These results show that carotenogenesis in S. faecium UNH564P is dependent on cultural conditions and medium glucose concentration. Carotenogenesis commenced at the onset of bacterial growth and the total carotenoid content increased with age and paralleled cell growth to become constant in the stationary phase (Fig. I). It was also observed that the total extractable lipid decreased in late-stationary phase. Such a phenomenon has been observed by others, for example in membrane fractions from Sarcina aurantiaca (Thirkell \& Gray, 1974), and it may reflect either active metabolism of the lipid or the formation of complexes with carbohydrate or protein in late-stationary growth phase. In this context, it should be noted that the relatively low levels of extractable lipid reported here in Streptococcus faecium (e.g. $0.57 \%$ of the dry weight of cells grown for $24 \mathrm{~h}$ on a medium containing $0.25 \%$ glucose) reflect the extraction method used, which was developed primarily for isolating non-saponifiable rather than total lipid. Extraction of S. faecium ( $24 \mathrm{~h}$ culture on a medium containing $0.25 \%$ glucose) with chloroform-methanol showed the lipid content to be $5.86 \%$ of the dry weight (Taylor and Davies, unpublished data); this is more likely to be an accurate estimate of the total lipid of the organism.

In general, no large alterations of the carotene and xanthophyll levels occurred as growth time was extended from 24 to $48 \mathrm{~h}$, but the carotene: xanthophyll ratio in static cultures was extremely sensitive to changes in the medium glucose concentration (Table I). The changes in this ratio may be related to the production of acid by the bacteria; bacterial growth was less than maximal at concentrations of glucose in excess of $0.50 \%$ and culture $\mathrm{pH}$ decreased with increasing glucose concentration but maintained a steady value $(4 \cdot 2)$ at concentrations above $0.75 \%$ (see Fig. 2). Total carotenoid production by the static cultures (Table I) was very low when the medium contained only $0.10 \%$ glucose, but much more pigment was 


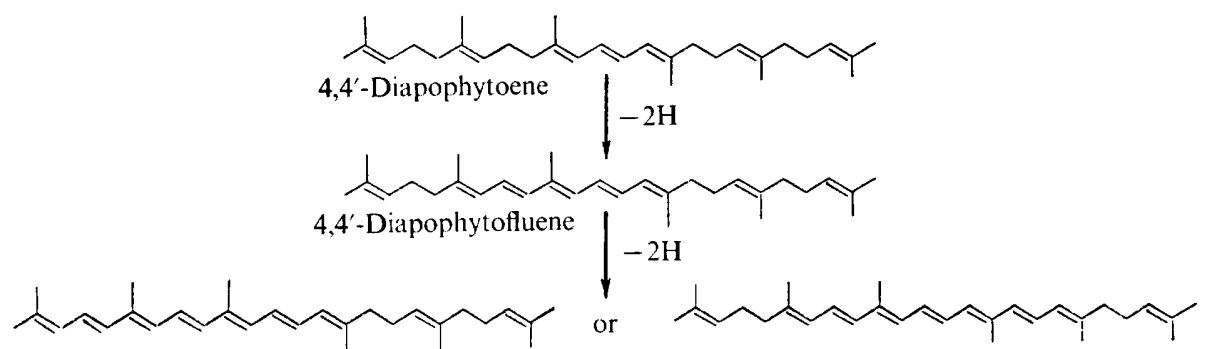

4,4'-Diapo-7,8,11,12-tetrahydrolycopene

$4,4^{\prime}$-Diapo- $\zeta$-carotene

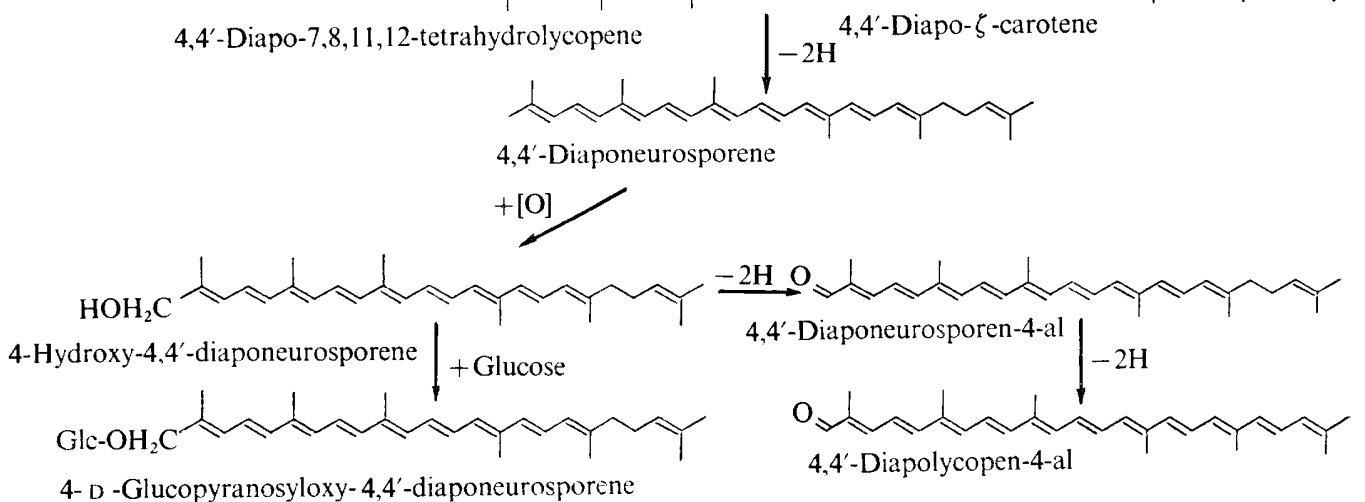

4- D -Glucopyranosyloxy-4,4'-diaponeurosporene

Fig. 3. Postulated pathway for the biosynthesis of triterpenoid carotenoids in S. faecium UNH564P.

formed when the glucose concentration was increased to $0.25 \%$, and maximum levels were attained with a total glucose supplement of $0.50 \%$. It appears that both carotenogenesis and cell growth in $S$. faecium are related to the availability of glucose, the latter linearly up to a critical $\mathrm{pH}$ of approximately $5^{\circ}$ (corresponding to a glucose concentration of about $0.35 \%$ ), above which both are partially inhibited. Bacterial growth, like carotenogenesis, appears to be optimal at a glucose concentration of $0.50 \%$ (Fig. 2). These observations are also consistent with the established view that the carbon: nitrogen $(C: N)$ ratio in the growth medium is an important factor in controlling carotenogenesis in micro-organisms (Goodwin, 1952). The amount of carotenoid formed increased with $C: N$ ratio, but only until other factors, such as acid production by the organism, exerted a limiting effect.

These studies also support the triterpenoid carotenoid biosynthetic pathway postulated for S. faecium and illustrated in Fig. 3. 4,4'-Diapophytoene is converted, by three successive didehydrogenations, into the most unsaturated carotene of the organism, 4,4'-diaponeurosporene. These dehydrogenations are obviously favoured in static cultures by higher concentrations of glucose; an increase in glucose concentration from 0.10 to $0.50 \%$ changed the proportions of diapophytoene and all-trans diaponeurosporene in the total carotenoid of $24 \mathrm{~h}$ static cultures from 85 and $2 \%$ respectively to 30 and $4 \mathrm{I} \%$ (Table 2). 4,4'-Diaponeurosporene is thought to be the substrate for a 4 -mono-oxygenase-catalysed formation of the primary allylic alcohol, 4 -hydroxy-4,4'-diaponeurosporene $\left(4,4^{\prime}\right.$-diapo- $7^{\prime}, 8^{\prime}$-dihydro$\psi, \psi$-caroten-4-ol; Taylor \& Davies, 1974b). This alcohol is converted primarily into its glucoside but also, by oxidative side-reactions which may be the beginning of a degradation process, into its corresponding aldehyde, 4,4'-diaponeurosporen-4-al (4,4'-diapo-7', $8^{\prime}$ -

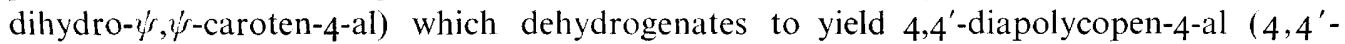
diapo- $y^{\prime}, \psi^{\prime}$-caroten-4-al; Taylor \& Davies, 1976). The formation by static cultures of all these xanthophylls, and of the glucoside in particular, was reduced at higher glucose concentrations (Table 2). 
The highly aerobic conditions produced by rapidly shaking $S$. faecium cultures resulted in dramatic changes in carotenoid content (Table 2). In contrast to the conclusion of other workers that a high aeration (of a halophilic coccus; Aasen, Francis \& Liaaen-Jensen, 1969) led to the increased production of some of the normal xanthophylls, our studies show that aeration of $S$. faecium cultured on a $0.25 \%$ glucose medium produced mainly carotenoid degradation products. It may be that aeration of aerobes leads to the production of normal carotenoids, but increasing the oxygen tension in cultures of facultative anaerobes (e.g. S. faecium) results rather in carotenoid degradation. Aeration of $S$. faecium cultures at a higher glucose concentration $(0.50 \%)$ led to the formation of a high proportion of 4-D-glucopyranosyloxy-4,4'-diaponeurosporene, the major xanthophyll of static cultures. The apparent sparing effect of a high glucose concentration on this xanthophyll may result from either a conversion of an easily oxidized, and therefore labile, xanthophyll (4-hydroxy$4,4^{\prime}$-diaponeurosporene ?) to its more stable glucoside (Fig. 3), or an inhibition of carotenoid oxidation, a process crucial to the postulated role of carotenoids as preferred substrates in protecting non-photosynthetic bacteria from otherwise lethal photo-induced oxidations (Krinsky, 197I).

We thank the Science Research Council for a research grant, and Dr W. R. Chesbro for providing the experimental organism.

\section{REFERENCES}

Aasen, A. J., Francis, G. W. \& Lianen-Jensen, S. (1969). Bacterial carotenoids. XXIX. The carotenoids of two yellow halophilic cocci - including a new glycosidic methyl apo-lycopenoate. Acta chemica scandinavica 23, 2605-26I 5 .

FopPen, F. H. (1969). On some pigments of Epicoccum nigrum Link. Annali dell'Istituto superiore di sanità, Roma 5, 439-5 3 .

Goodwin, T. W. (1952). The Comparative Biochemistry of the Carotenoids. London: Chapman \& Hall.

Goodwin, T. W. (1955). Carotenoids. Annual Review of Biochemistry 24, 497-522.

Goodwin, T. W. (1956). The carotenoids of photosynthetic bacteria. II. The carotenoids of a number of non-sulphur purple photosynthetic bacteria (Athiorhodaceae). Archiv für Mikrobiologie 24, 31 3-322.

Goodwin, T. W. (197I ). Algal carotenoids. In Aspects of Terpenoid Chemistry and Biochemistry, pp. 3I 5-356. Edited by T. W. Goodwin. London and New York: Academic Press.

Krinsky, N. I. (197I ). Function. In Carotenoids, pp. 669-7I6. Edited by O. Isler. Basel: Birkhäuser.

van Niel, C. B., Goodwin, T. W. \& Sissins, M. E. (1956). Studies in carotenogenesis. XXI. The nature of the changes in carotenoid synthesis in Rhodospirillum rubrum during growth. Biochemical Journal $\mathbf{6 3}_{3}$, $408-412$.

Razin, S. \& Cleverdon, R. C. (1965). Carotenoids and cholesterol in membranes of Mycoplasma laidlawii. Journal of General Microbiology 4I, 409-4I 5.

RaZin, S. \& RotTem, S. (1967). Role of carotenoids and cholesterol in the growth of Mycoplasma laidlawii. Journal of General Microbiology $93, \mathrm{I}$ 181-I 182.

TAYloR, R. F. \& Davies, B. H. (1974a). Triterpenoid carotenoids and related lipids: the triterpenoid carotenes of Streptococcus faecium UNH 564P. Biochemical Journal 139, 75I-760.

TAYlor, R. F. \& Davies, B. H. (1974b). Triterpenoid carotenoids and related lipids: triterpenoid monohydroxy- and monoglucosyloxy-carotenoids from Streptococcus faecium UNH $564 \mathrm{P}$. Biochemical Journal I39, 76I-769.

TAYloR, R. F. \& Davies, B. H. (1976). Triterpenoid carotenoids and related lipids: triterpenoid carotenoid aldehydes from Streptococcus faecium UNH 564P. Biochemical Journal (in the Press).

Thirkell, D. \& Gray, E. M. M. (I974). Chemical composition of a purified membrane fraction from Sarcina aurantiaca in relation to growth phase. Antonie van Leeuwenhoek 40, 65-70. 